Published in final edited form as:

Cochrane Database Syst Rev. ; 2014: . doi:10.1002/14651858.CD011075.

\title{
Acupuncture for acute hordeolum
}

\author{
Ke Cheng ${ }^{1}$, Xue Wang ${ }^{2}$, Menghu Guo ${ }^{1}$, L. Susan Wieland ${ }^{3}$, Xueyong Shen ${ }^{4}$, and Lixing \\ Lao $^{5}$ \\ ${ }^{1}$ College of Acupuncture-Moxibustion and Tuina, Shanghai University of Traditional Chinese \\ Medicine, Shanghai, China \\ 2Department of Epidemiology, Johns Hopkins Bloomberg School of Public Health, Baltimore, \\ Maryland, USA \\ ${ }^{3}$ Center for Evidence-based Medicine, Brown University Public Health Program, Providence, \\ Rhode Island, USA \\ ${ }^{4}$ College of Acupuncture-Moxibustion and Tuina, Shanghai University of Traditional Chinese \\ Medicine, Shanghai Research Center of Acupuncture \& Meridians, Shanghai, China \\ ${ }^{5}$ School of Chinese Medicine, The University of Hong Kong, Hong Kong, China
}

\section{Abstract}

This is the protocol for a review and there is no abstract. The objectives are as follows:

The objective of this review is to determine the effects and, when possible, the safety of acupuncture for the treatment of acute hordeola, in comparison to no specific treatment (e.g., observation), sham acupuncture, or other active treatments. Acupuncture as an adjuvant to another treatment also will be compared to that treatment alone.

\section{BACKGROUND}

\section{Description of the condition}

\begin{abstract}
A hordeolum is a common disorder of the eyelid (Ehrenhaus 2012; Skorin 2002). It is an acute, red, painful, and localized inflammation with abscess formation, usually caused by obstructed orifices of the sebaceous glands of the eyelid. The infected sebaceous glands can be the meibomian gland in the tarsal plate (internal hordeolum) or the glands of Zeis or Moll associated with eyelash follicles (external hordeolum, or stye) (Barza 1983; Chern 2011; Gerstenblith 2012; Grant 2013; Mueller 2008; Peralejo 2008; Tasman 2013). An episode is usually self-limited and may resolve in about a week with spontaneous drainage of the
\end{abstract}

Copyright $(92014$ The Cochrane Collaboration. Published by John Wiley \& Sons, Ltd.

Contact address: Xueyong Shen, College of Acupuncture-Moxibustion and Tuina, Shanghai University of Traditional Chinese Medicine, Shanghai Research Center of Acupuncture \& Meridians, 1200, Cailun RD, Shanghai, 201203, China. snowysh@hotmail.com.

\section{CONTRIBUTIONS OF AUTHORS}

$\mathrm{KC}$ and XS conceived and designed the topic; KC, XW, and MG contributed to writing the protocol; all of the authors commented on drafts of the protocol and assisted with preparing responses to CEVG editorial base and peer review comments.

DECLARATIONS OF INTEREST

None known. 
abscess (Chern 2011; Deibel 2013; Lederman 1999; Peralejo 2008; Skorin 2002; Wald 2007). When the condition is severe, it is sometimes accompanied by fever or chills (Garrity 2007), and the inflammation may spread to other adjacent glands and tissue (Skorin 2002).

Recurrences are also common (Garrity 2007; Skorin 2002). An untreated internal hordeolum may develop into a chalazion or generalized eyelid cellulitis (Bessette 2012; Chern 2011; Deibel 2013; Ehrenhaus 2012; Gerstenblith 2012; Lederman 1999).

Hordeola are among the most common eyelid lesions seen in clinical practice, but no data are available on the precise incidence and prevalence (Ehrenhaus 2012). Hordeola are more common in adults than in children, but are not limited to any age, gender, or racial group (Ehrenhaus 2012; Lindsley 2013). The usual cause of an acute hordeolum is the Staphylococcus aureus bacterium (Bessette 2012; Chern 2011; Ehrenhaus 2012; Gerstenblith 2012; Mueller 2008; Peralejo 2008; Tasman 2013; Wald 2007). Recent studies have reported that about $90 \%$ of hordeola are associated with S. aureus (Bharathi 2010) and that $24 \%$ of methicillin-resistant $S$, aureus ocular infections are eyelid disorders including hordeola (Hsiao 2012). Individuals with poor eyelid hygiene, inflammatory diseases of the eyelid (e.g., blepharitis, meibomitis, or rosacea), and stress, or experiencing hormonal changes are at greater risk of developing a hordeolum than the general population (Bessette 2012; Grant 2013).

Conservative treatment measures include warm compresses several times a day, which may help drainage. Topical antibiotics also may be prescribed by the ophthalmologist. If these fail, incision and drainage may be performed and systemic antibiotics are indicated (Chern 2011; Gerstenblith 2012; Lindsley 2013; Mueller 2008; Peralejo 2008; Tasman 2013).

\section{Description of the intervention}

According to the philosophy of traditional acupuncture, energy (i.e., $q i$ and blood in traditional Chinese medicine (TCM)) circulates in 'meridians' located throughout the body. When the meridian energy circulation is blocked by certain pathogenic factors, pain or ill health will result. The way to restore energy circulation, health, and balance is to stimulate the appropriate combination of the estimated 400 traditional meridian acupuncture points in the body (WHO 1991) by acupuncture. According to TCM literature and clinical practice, acupuncture is effective for many kinds of eye diseases (Cheng 2010; Shen 2007). A recent systematic review also found some limited evidence for the effectiveness of acupuncture in the treatment of dry eye syndrome (Lee 2011). For other eye disorders, such as glaucoma and myopia, systematic reviews have drawn no conclusions regarding the benefit of acupuncture, mainly because of the sparse data (Law 2013; Wei 2011). Acupuncture, in a broader sense, includes several kinds of techniques (e.g., traditional body needling, electroacupuncture, moxibustion, etc.) (WHO 2003). According to TCM principles, redness, swelling, and pain of the eyelid are caused by pathogenic "wind heat" causing $q i$ and blood stagnation at the eyelid (Shen 2007). Hence, the acupoints with the action of clearing heat are those usually used in treatment (i.e., stimulated by either needle insertion, acupressure, or bloodletting, etc). Bloodletting with a three-edged needle is used more often than other acupuncture techniques in clearing "heat", reducing swelling, and alleviating pain, and thus is the most common technique used in treating an acute hordeolum. Bloodletting for the 
treatment of an acute hordeolum involves the withdrawal of a very small quantity of blood, and the most commonly used points are Erjian (EX-HN6) at the ear apex and Taiyang (EXHN5) at the temporal region (Shen 2007).

\section{How the intervention might work}

So far, few studies have explored the mechanism of acupuncture treatment for acute hordeola. The mechanism of acupuncture analgesia has been studied most widely. Different mechanisms of action have been proposed for the biological basis of acupuncture analgesia. For example, animal studies have provided evidence that acupuncture stimulates the release of neurochemicals (usually endogenous opioids or serotonin) (Han 1980; Han 2003). 'Gate theory' is another proposed mechanism for acupuncture analgesia (i.e., stimulation by the acupuncture needles may suppress the nervous system pathway of nociceptive pain signals (Man 1972)). Some studies have found that acupuncture may affect the autonomic nervous system that regulates involuntary body functions such as immune reactions and the processes that govern blood pressure, blood flow, and body temperature (Moffet 2006). Basic science studies also show that acupuncture suppresses inflammation (Li 2008), the main symptom of an acute hordeolum.

\section{Why it is important to do this review}

The most current Cochrane review of interventions for acute internal hordeola (Lindsley 2013) found that none of the most commonly used conventional treatments, including heated compresses and antibiotics or steroids, could be evaluated systematically due to the lack of randomized controlled trials (RCTs) investigating these treatments. In addition, no RCTs evaluating surgical interventions, such as incision and curettage, were identified. However, during the literature search for the Lindsley 2013 review, a number of RCTs on acupuncture for acute hordeola were identified, reflecting the popularity of this treatment and implying the need for a systematic review on acupuncture for this condition. Acupuncture has been demonstrated to be a safe treatment with very low risk of serious side effects (Cherkin 2003; Xu 2013). Four large, prospective surveys of acupuncture practitioners (MacPherson 2001; Melchart 2004; White 2001; Witt 2009) confirmed that serious adverse events after acupuncture are uncommon. Acupuncture for the treatment of an acute hordeolum is relatively convenient, usually comprising three or fewer sessions, with each session lasting for a few minutes only, which indicates its applicability in the clinic.

Given the existing evidence, a systematic review of the effectiveness and safety of acupuncture for the treatment of acute hordeola is highly desirable.

\section{OBJECTIVES}

The objective of this review is to determine the effects and, when possible, the safety of acupuncture for the treatment of acute hordeola, in comparison to no specific treatment (e.g., observation), sham acupuncture, or other active treatments. Acupuncture as an adjuvant to another treatment also will be compared to that treatment alone. 


\section{METHODS}

\section{Criteria for considering studies for this review}

Types of studies-We will include RCTs.

A study in 2009 indicated that most of the reports of RCTs in Chinese journals lacked an adequate description of randomization, and more than $90 \%$ of them did not adhere to accepted methodology for randomization (Wu 2009). A more recent study reported that only one of 14 Chinese-language studies labeled as RCTs could be confirmed to be an RCT (Adams 2012). We will therefore contact the authors of RCTs published in Chinese journals that claim to have randomized participants but have not included details about the randomization methods in their publications in order to determine whether they used proper methods for random sequence generation. The interviews will be conducted using questions adapted from the survey developed by $\mathrm{Wu} 2009$. We also will contact the authors of nonChinese-language RCTs whenever their reports do not include details about randomization methods.

Types of participants-We will include studies of participants with acute hordeola, including acute internal hordeola and acute external hordeola (styes). Studies of participants with chronic hordeola or chalazia will be excluded.

Types of interventions-We will include RCTs evaluating TCM acupuncture. Acupuncture methods used for treating acute hordeola include, but are not limited to, body needle insertion on acupoints; bloodletting at Erjian (EX-HN6) at the ear apex or at Taiyang (EX-HN5) at the temporal region; pricking or bloodletting at Arshi points (i.e., reflection spots that are small pink pustules or papules) at the regions of the thoracic vertebra between the scapulae; acupressure; and electroacupuncture. We will exclude RCTs of laser acupuncture and noninvasive electrostimulation because, in contrast to conventional needle acupuncture or acupressure, neither of these techniques involves the mechanical stimulation of acupoints, and hence are considered different interventions. We will exclude trials of injection acupuncture, which involves the injection of a herb, drug, or vitamin at the acupuncture point, because this procedure is a completely different intervention to needle acupuncture. However, as acupuncture is often accompanied by moxibustion, we will include trials using moxibustion as a cointervention with acupuncture.

We will include RCTs that compare acupuncture to no specific treatment (observation), sham acupuncture, or an active conventional treatment (e.g., hot or warm compresses, lid scrubs, antibiotics, or steroids), or that compare acupuncture as an adjuvant to another treatment to the other treatment alone. Adjuvant treatments, either Western medicine (e.g., antibiotics) or TCM (e.g., intake of traditional Chinese herbal medicine), will be allowed as long as they have been given to both the acupuncture and control groups.

\section{Types of outcome measures}

Primary outcomes: The primary outcome for the comparison of treatments will be the proportion of participants with complete resolution of a hordeolum at short-term follow up (i.e., less than or equal to seven days and closest to seven days after diagnosis). We chose 
the time closest to seven days after diagnosis as a criterion in case a study reports the outcome at multiple time points. The seven-day period for resolution is selected because most cases of hordeola resolve on their own between one and two weeks.

Secondary outcomes: Secondary outcomes for the comparison of treatments will include:

- the proportion of participants with complete resolution of the hordeolum at longerterm follow-up (i.e., 8 to 30 days and closest to 14 days after diagnosis);

- the proportion of participants requiring surgical incision and drainage after the treatment period or seven days after diagnosis;

- the proportion of participants with occurrence of a chalazion after the treatment period or seven days after diagnosis;

- the proportion of participants with recurrence of a hordeolum after six months and one year (a recurrence is defined as any hordeolum that occurs after one month from the resolution of the initial hordeolum and at any location on the same eyelid, or as defined by the included study);

- the proportion of participants with a secondary hordeolum during or after the treatment period and seven days after diagnosis (a secondary hordeolum is defined as a hordeolum that occurs within one month of the initial hordeolum and at a different location than the initial hordeolum, or as defined by the included study).

Adverse outcomes: We plan to document all adverse effects related to the treatment of a hordeolum that are reported in the primary studies during and after the treatment period. Specific adverse outcomes of interest will include, but are not limited to, conjunctivitis; eye irritation; discoloration of the eyelid, conjunctiva, or lens; and corneal damage. The specific adverse outcomes of bloodletting will include infection at the local point where the acupuncture was applied (Xu 2013). We plan to assess adverse outcomes at longer-term follow up (i.e., 8 to 30 days and closest to 14 days after diagnosis).

Economic outcomes: We plan to document any economic outcome data, such as cost of acupuncture, as reported in the included studies, at longer-term follow-up (i.e., 8 to 30 days and closest to 14 days after diagnosis).

\section{Search methods for identification of studies}

Electronic searches-We will search the Cochrane Central Register of Controlled Trials (CENTRAL) (which contains the Cochrane Eyes and Vision Group Trials Register) (The Cochrane Library), MEDLINE (OVID), EMBASE, PubMed, Latin American and Caribbean Health Sciences Literature Database (LILACS), the meta Register of Controlled Trials ( $m$ RCT) (www.controlled-trials.com), ClinicalTrials.gov (www.clinicaltrials.gov), and the WHO International Clinical Trials Registry Platform (ICTRP) (www.who.int/ictrp/ search/en). We will also search the Chinese databases Sino-Med (previously called the Chinese Biomedical Database, http://www.sinomed.ac.cn/), Chinese National Knowledge Infrastructure (http://www.cnki.net/), and VIP Database for Chinese Technical Periodicals 
(http://lib.cqvip.com/). We will not use any date or language restrictions in the electronic searches.

See: Appendices for details of search strategies for CENTRAL (Appendix 1), MEDLINE (Appendix 2), EMBASE (Appendix 3), LILACS (Appendix 4), PubMed (Appendix 5), Chinese CNKI Database (Appendix 6), SinoMed Database (Appendix 7), Chinese VIP Database (Appendix 8), $m$ RCT (Appendix 9), Clinical-Trials.gov (Appendix 10) and the ICTRP (Appendix 11).

Searching other resources-We will review the reference lists from potentially eligible studies to identify further studies. We will not employ handsearching for the specific purposes of this review.

\section{Data collection and analysis}

Selection of studies-Two authors will independently screen all records identified by the searches. According to the inclusion criteria, each review author will assess titles and abstracts as 'definitely relevant', 'possibly relevant', or 'definitely not relevant'. We will resolve discrepancies by discussion and will then obtain the full-text copies of reports assessed as 'definitely relevant' or 'possibly relevant' by both review authors. Two authors will then independently label each study as 'include' or 'exclude'. We will resolve disagreements by discussion. We will document the reasons for studies labeled as 'excluded' by both review authors in the 'Characteristics of excluded studies' table. We will assess studies for which the randomization methods are not clearly described and for which author contact details could not be located, or for which the authors did not respond to interview requests as 'Studies awaiting classification'. We will set the response time at two weeks and will document any communications with study authors. We will assess the risk of bias for all the included studies.

Data extraction and management-Two review authors will independently extract data using the data extraction forms created by the Cochrane Eyes and Vision Group (CEVG). For each included study, we will extract data on study design, participant characteristics, interventions, outcomes, and cost, as well as other relevant information. We will pilot test the extraction form for all aspects of the data collection on a sample of included studies, when possible, prior to the full extraction of all studies in order to ensure a similar extraction approach across review team members. One review author will enter the data into Review Manager 5.2 (RevMan 2013) and a second review author will verify the data entry. Discrepancies between review authors will be resolved by discussion or consultation with a third review author.

When reported data are incomplete or ambiguous, we will request additional information or clarification from the corresponding study author. If we do not receive a response in two weeks, we will include the data as available in the study report(s).

Adequacy of treatment: Two acupuncturists (XS and LL), who have a combined clinical experience of nearly 50 years in acupuncture, and who have previously worked on RCTs and systematic reviews of acupuncture, will independently assess the adequacy of the 
acupuncture administered in the trials. Six aspects of the acupuncture intervention will be assessed for adequacy: 1) choice of acupuncture points; 2) total number of sessions; 3 ) treatment duration; 4) treatment frequency; 5) acupuncture technique; and 6) acupuncturist's experience (Manheimer 2010). The likelihood of the sham intervention having physiological activity will also be assessed using an open-ended question on the data abstraction form, such as "What are your opinions or comments about the sham or placebo intervention?". The control groups also will be assessed for: 1) appropriateness of sham/placebo intervention; and 2) adequate number of sessions/dose (for the active treatments used in control group). The acupuncturists will be provided with only the part of each publication that describes the acupuncture and control procedures, so that their assessments cannot be influenced by the results of the trials. To test the success of masking the acupuncturist to the study publication and results, we will ask the assessors to guess the identity of each RCT being assessed. The acupuncturists will assess adequacy independently and will achieve consensus by discussion.

Assessment of risk of bias in included studies-Two review authors will independently assess the risk of bias in included studies based on the methods provided in Chapter 8 of the Cochrane Handbook for Systematic Reviews of Interventions (Higgins 2011a). As recommended, we will use the following seven separate criteria:

- adequate sequence generation;

- allocation concealment;

- masking (blinding) of participants and study personnel;

- masking of outcome assessors;

- incomplete outcome data and percentage of missing data among treatment groups;

- $\quad$ selective outcome reporting; and

- other potential sources of bias.

For 'other potential sources of bias', we plan to assess the following items:

- groups similar at baseline with regard to the most important prognostic indicators;

- cointerventions avoided or similar between intervention groups;

- compliance acceptable in all groups;

- timing of the outcome assessment similar in all intervention groups; and

- funding sources and conflicts of interest.

We will assess the risk of bias for different outcomes based on whether masking of outcome assessors was performed and whether completeness of outcome data were reported consistently, where applicable. For each study included in the review, we will judge each criterion to have low, unclear, or high risk of bias. Discrepancies between review authors will be resolved by a third review author. 


\section{Measures of treatment effect}

Dichotomous data: The primary outcome of interest, the proportion of participants with complete resolution of a hordeolum at short-term follow up (i.e., less than or equal to seven days and closest to seven days after diagnosis), will be analyzed as a dichotomous variable: resolved versus not resolved. Therefore, in cases where outcomes, such as an improvement in an acute hordeolum, are presented in the form of an ordinal outcome (e.g., resolved, improved, or ineffective), we will create a dichotomous measure in which the outcomes other than complete resolution (e.g., improved and ineffective) are combined as negative category (Deeks 2011). Data on the proportion of participants requiring surgical incision and drainage after treatment, the proportion of participants developing a chalazion after treatment, the proportion of participants with recurrent hordeola, and the proportion of participants with secondary hordeola will also be analyzed as dichotomous data. We will report dichotomous data as a summary risk ratio with its $95 \%$ confidence interval.

Counts and rate data: We will summarize counts and rate data as rate ratios (e.g., hazard ratios) when the event is rare, and as continuous outcome data when the event is more common. We will analyze adverse event data as counts and rates. We will report continuous data as summary mean differences with $95 \%$ confidence intervals.

Unit of analysis issues-The unit of analysis for this review will be the individual participant, not an eyelid of an individual. The reason for this is that, according to the TCM principle of acupuncture treatment for an acute hordeolum (i.e., clearing pathogenic "wind heat" in the body is a whole body effect), the effect of acupuncture is usually not limited to a specific eye (Cheng 2010; Shen 2007). We will therefore exclude from our review any trial in which participants had one eye randomized to acupuncture and the other eye to control.

Dealing with missing data-For our primary analyses, we will exclude participants with unrecorded resolution data and use only the data available.

Assessment of heterogeneity-We will evaluate clinical and methodological heterogeneity in terms of study characteristics, participant inclusion/exclusion criteria, and primary and secondary outcomes. We will assess statistical heterogeneity using summary test statistics ( $\mathrm{I}^{2}$ statistic). When the $\mathrm{I}^{2}$ statistic is greater than or equal to $50 \%$, the summary measures of effect will be interpreted with caution. We also will examine the $\mathrm{Chi}^{2}$ statistic for heterogeneity and the degree of overlap in confidence intervals of included studies. Poor overlap may suggest the presence of heterogeneity.

Assessment of reporting biases-When there are at least 10 studies included in a meta-analysis, we will examine the funnel plot symmetry for the analysis in order to assess the potential for publication bias. We will assess the possibility of selective outcome reporting for each study as part of the 'Risk of bias' assessment.

Data synthesis-We will combine data from individual RCTs in meta-analysis when the RCTs are sufficiently similar in terms of the following study characteristics.

\section{Control group:}


a. no specific treatment (observation);

b. sham acupuncture;

c. other active treatment; and

d. other treatment alone (compared with acupuncture as an adjuvant to the other treatment).

\section{Outcome measures:}

a. complete resolution of a hordeolum at short-term follow up (i.e., less than or equal to seven days and closest to seven days after diagnosis);

b. complete resolution of a hordeolum at longer-term follow up (i.e., 8 to 30 days and closest to 14 days after diagnosis);

c. requiring surgical incision and drainage after the treatment period or seven days after diagnosis;

d. occurrence of a chalazion after the treatment period or seven days after diagnosis;

e. recurrence of a hordeolum after six months after diagnosis;

f. recurrence of a hordeolum after one year after diagnosis;

g. occurrence of a secondary hordeolum during or after the treatment period and seven days after diagnosis;

h. adverse events; and

i. economic data

When limited statistical heterogeneity is suggested (defined here as $\mathrm{I}^{2}<50 \%$ ), we will pool data using the random-effects model because of the expected heterogeneity among the studies' acupuncture protocols and settings, unless there are fewer than three trials, in which case we will use the fixed-effect model. When substantial heterogeneity is detected, we will combine trial results by relevant, less heterogeneous subgroups if sufficient data are available. Otherwise we will describe the results individually.

Regardless of whether there is sufficient information available to use quantitative analyses to summarize the data, we will assess the overall quality of the evidence for each outcome. To accomplish this, we will use the GRADE (Grades of Recommendation, Assessment, Development and Evaluation) approach, as recommended in Chapter 12 of the Cochrane Handbook for Systematic Reviews of Interventions (Schünemann 2011). Factors that may decrease the quality of the evidence are: study design and risk of bias; inconsistency of results; indirectness (not generalizable); imprecision (sparse data); and other factors (e.g., reporting bias). The quality of the evidence for a specific outcome will be reduced by a level according to the performance of the studies against these five factors.

High-quality evidence: there are consistent findings among at least 75\% of RCTs with a low risk of bias; consistent, direct, and precise data; and no known or suspected publication 
biases. Further research is unlikely to change either the estimate or our confidence in the results.

Moderate-quality evidence: one of the criteria is not met. Further research is likely to have an important impact on our confidence in the estimate of effect and may change the estimate.

Low-quality evidence: two of the criteria are not met. Further research is very likely to have an important impact on our confidence in the estimate of effect and is likely to change the estimate.

Very-low-quality evidence: three of the criteria are not met. We are very uncertain about the results.

No evidence: no RCTs were identified that addressed this outcome.

Subgroup analysis and investigation of heterogeneity-We will conduct subgroup analysis on one clinical characteristic that may influence the effect of acupuncture on an acute hordeolum (i.e., the type of hordeolum: internal or external hordeolum).

Sensitivity analysis-We will conduct sensitivity analyses specific to the primary outcome by imputing data for the proportion of participants with complete resolution for the missing participants using the following three methods: 1) impute data using the proportion of participants with complete resolution observed in the control group; 2 ) impute data using the proportion of participants with complete resolution among completers in the separate groups (i.e., the acupuncture group and the control group, respectively); and 3) assume that all randomized participants with missing outcomes achieved a complete resolution (Higgins 2011b). We also will conduct sensitivity analyses to evaluate whether any meta-analysis results that are statistically significant remain statistically significant when we restrict metaanalysis to only trials judged to be at low risk on the following 'Risk of bias' criteria: allocation concealment; masking of participants (for sham- versus non-sham-controlled trials); masking of outcome assessors; and incomplete outcome data. We will conduct sensitivity analyses to determine the impact of studies assessed to have used inadequate treatment by excluding trials judged as 'inadequate' and 'unclear' on each of the six aspects of adequacy of treatment.

\section{Acknowledgments}

We thank Kristina Lindsley for her comments and suggestions during the title registration and her help in preparing responses to the CEVG editorial base. We also thank our peer reviewers, Tae-Young Choi, Wei Chen, and Barbara Hawkins, for their insightful comments for this protocol.

Richard Wormald (Co-ordinating Editor for CEVG) acknowledges financial support for his CEVG research sessions from the Department of Health (NIHR) through the award made by the National Institute for Health Research to Moorfields Eye Hospital NHS Foundation Trust and UCL Institute of Ophthalmology for a Specialist Biomedical Research Centre for Ophthalmology.

The views expressed in this publication are those of the authors and not necessarily those of the NIHR, National Health Service, or the Department of Health. 


\section{Appendix 1. CENTRAL search strategy}

\#1 MeSH descriptor: [Hordeolum] explode all trees

\#2 (Hordeol* or stye or styes)

\#3 MeSH descriptor: [Eye] explode all trees

\#4 sty

\#5 \#3 and \#4

\#6 MeSH descriptor: [Meibomian Glands] explode all trees

\#7 (Meibomian* adj3 (gland* or cyst* or infection* or inflammat*))

\#8 (tarsal adj3 (gland* or cyst* or infection* or inflammat*))

\#9 (palpebral adj3 (gland* or cyst* or infection* or inflammat*))

\#10 (conjunctiv* adj3 (gland* or cyst*))

\#11 (gland* adj5 (zeis* or Moll*))

\#12 (lid* or eyelid* or "eye margin") adj3 inflammat*

\#13 (lid* or eyelid* or "eye margin") adj3 infection*

$\# 14 \# 1$ or \#2 or \#4 or \#6 or \#7 or \#8 or \#9 or \#10 or \#11 or \#12 or \#13

\#15 MeSH descriptor: [Acupuncture] explode all trees

\#16 MeSH descriptor: [Acupuncture Therapy] explode all trees

\#17 MeSH descriptor: [Medicine, Chinese Traditional] explode all trees

\#18 MeSH descriptor: [Bloodletting] explode all trees

\#19 acupunctur*

\#20 (meridian* or moxi*)

\#21 (electrostimulat* or electroacupunctur*)

\#22 (electro* next/1 (stimulat* or acupunctur*))

\#23 acupoint*

\#24 (erjian or taiyang or EX-HN6 or EX-HN5)

\#25 body needl*

\#26 (bloodletting or blood-letting)

\#27 (Ear-apex or temporal region)

\#28 qi

\#29 ((chinese near/3 medicin*) or TCM)

$\# 30 \# 15$ or \#16 or \#17 or \#18 or \#19 or \#20 or \#21 or \#22 or \#23 or \#24 or \#25 or \#26 or \#27 or \#28 or \#29 
\#31 \#14 and \#30

\section{Appendix 2. MEDLINE (OVID) search strategy}

1. Randomized Controlled Trial.pt.

2. Controlled Clinical Trial.pt.

3. (randomized or randomised).ab,ti.

4. placebo.ab,ti.

5. drug therapy.fs.

6. randomly.ab,ti.

7. trial.ab,ti.

8. groups.ab,ti.

9. 1 or 2 or 3 or 4 or 5 or 6 or 7 or 8

10. exp animals/not humans.sh.

11. 9 not 10

12. exp hordeolum/

13. (Hordeol* or stye or styes).tw.

14. sty.tw.

15. exp eyes/

16. 14 and 15

17. exp meibomian glands/

18. (Meibomian* adj3 (gland* or cyst* or inflammat* or infection*)).tw.

19. (tarsal adj3 (gland* or cyst* or inflammat* or infection*)).tw.

20. (palpebral adj3 (gland* or cyst* or inflammat* or infection*)).tw.

21. (conjunctiv* adj3 (gland* or cyst*)).tw.

22. (gland* adj5 (zeis* or Moll*)).tw.

23. ((lid* or eyelid* or eye margin) adj3 inflammat*).tw.

24. ((lid* or eyelid* or eye margin) adj3 infection*).tw.

25. 12 or 13 or 16 or 17 or 18 or 19 or 20 or 21 or 22 or 23 or 24

26. 11 and 25

27. exp Acupuncture/

28. $\exp$ Acupuncture Therapy/

29. exp Medicine, Chinese Traditional/ 
30. acupunctur*.tw.

31. (meridian* or moxi*).tw.

32. (electrostimulat* or electroacupunctur*).tw.

33. (electro* adj1 (stimulat* or acupunctur*)).tw.

34. acupoint*.tw.

35. exp Bloodletting/

36. (erjian or taiyang or EX-HN6 or EX-HN5).tw.

37. body needl*.tw.

38. (bloodletting or blood-letting).tw.

39. (Ear-apex or temporal region).tw.

40. qi.tw.

41. ((chinese adj3 medicin*) or TCM).tw.

42. or $/ 27-41$

43. 26 and 42

The search filter for trials at the beginning of the MEDLINE strategy is from the published paper by Glanville et al (Glanville 2006).

\section{Appendix 3. EMBASE.com search strategy}

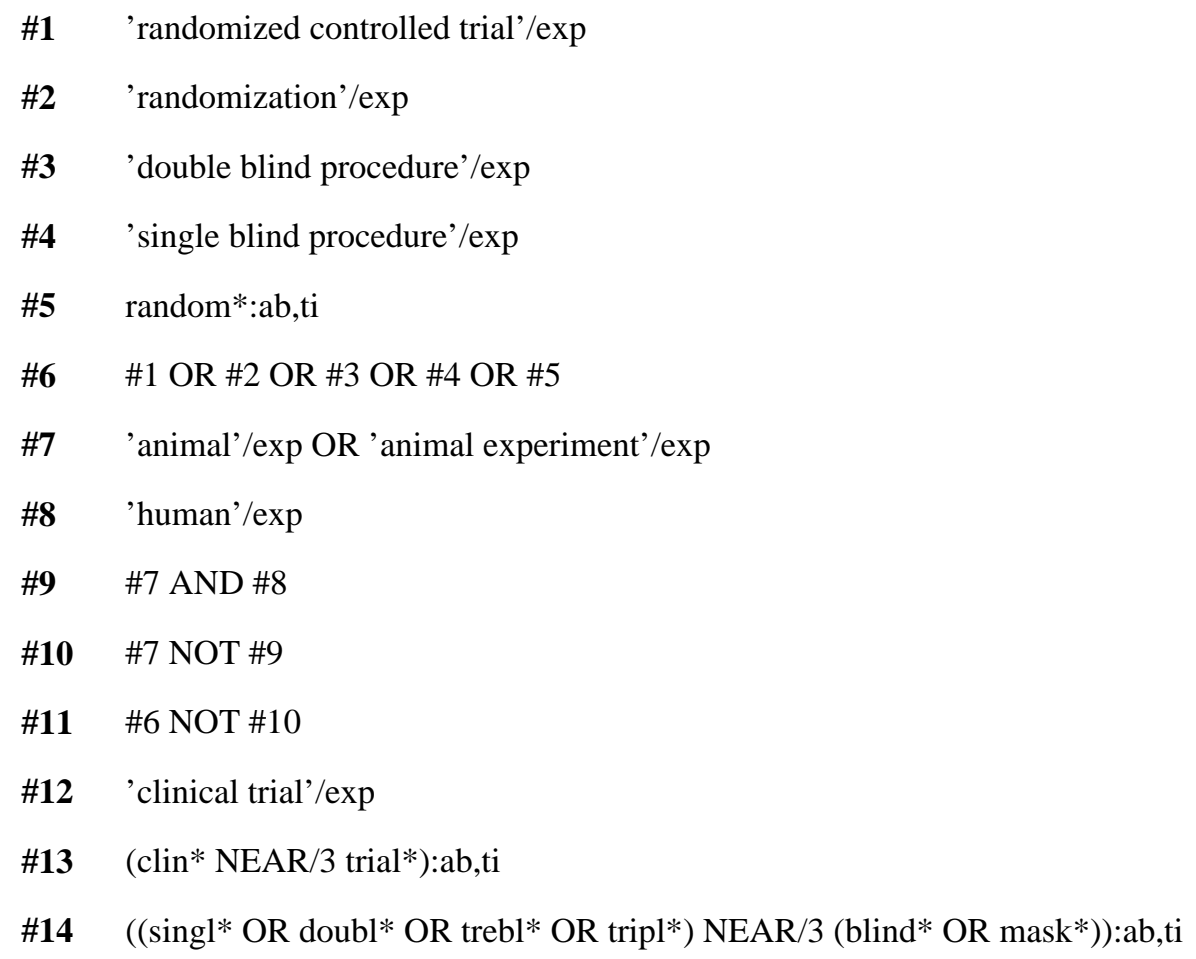




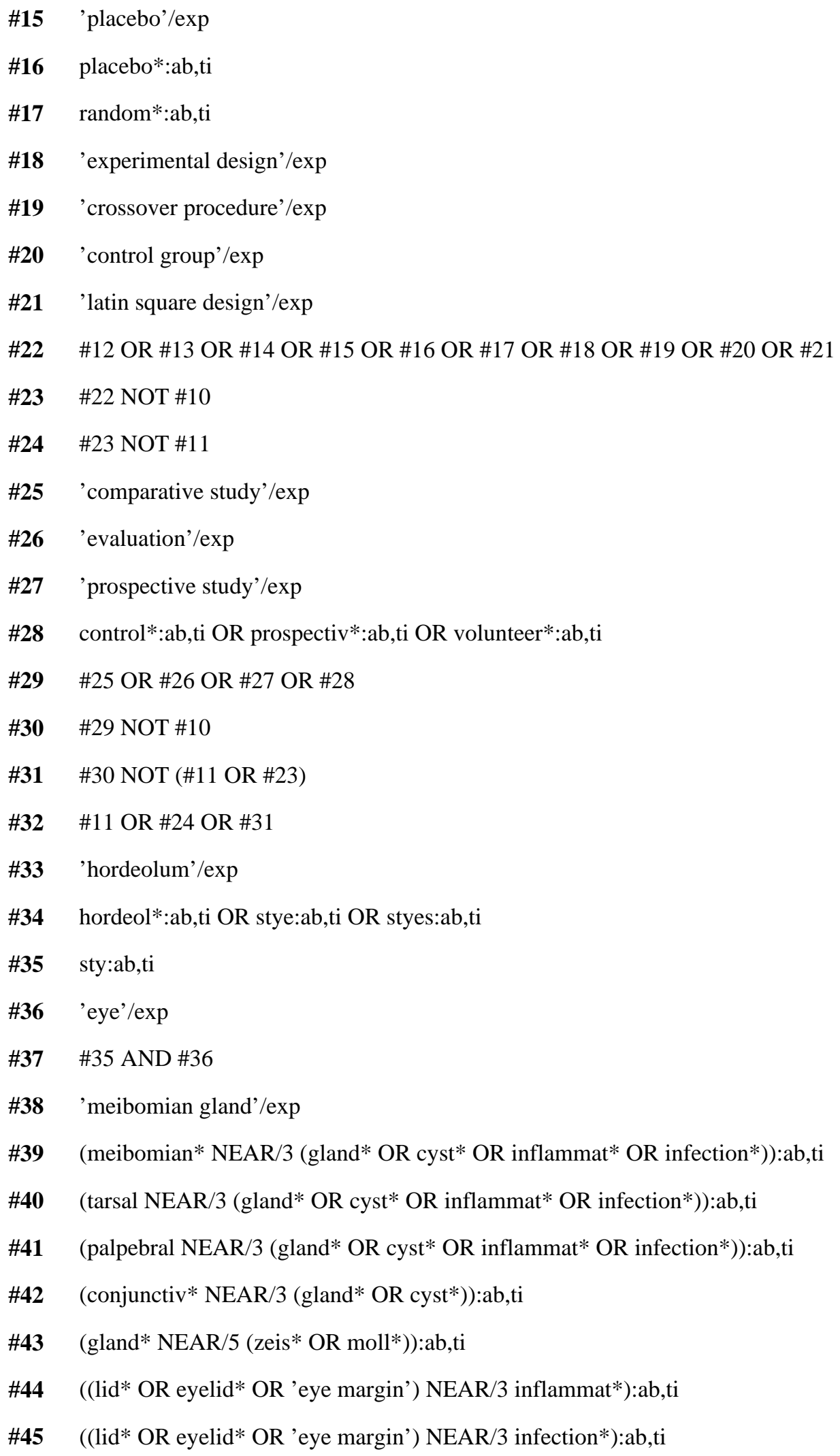




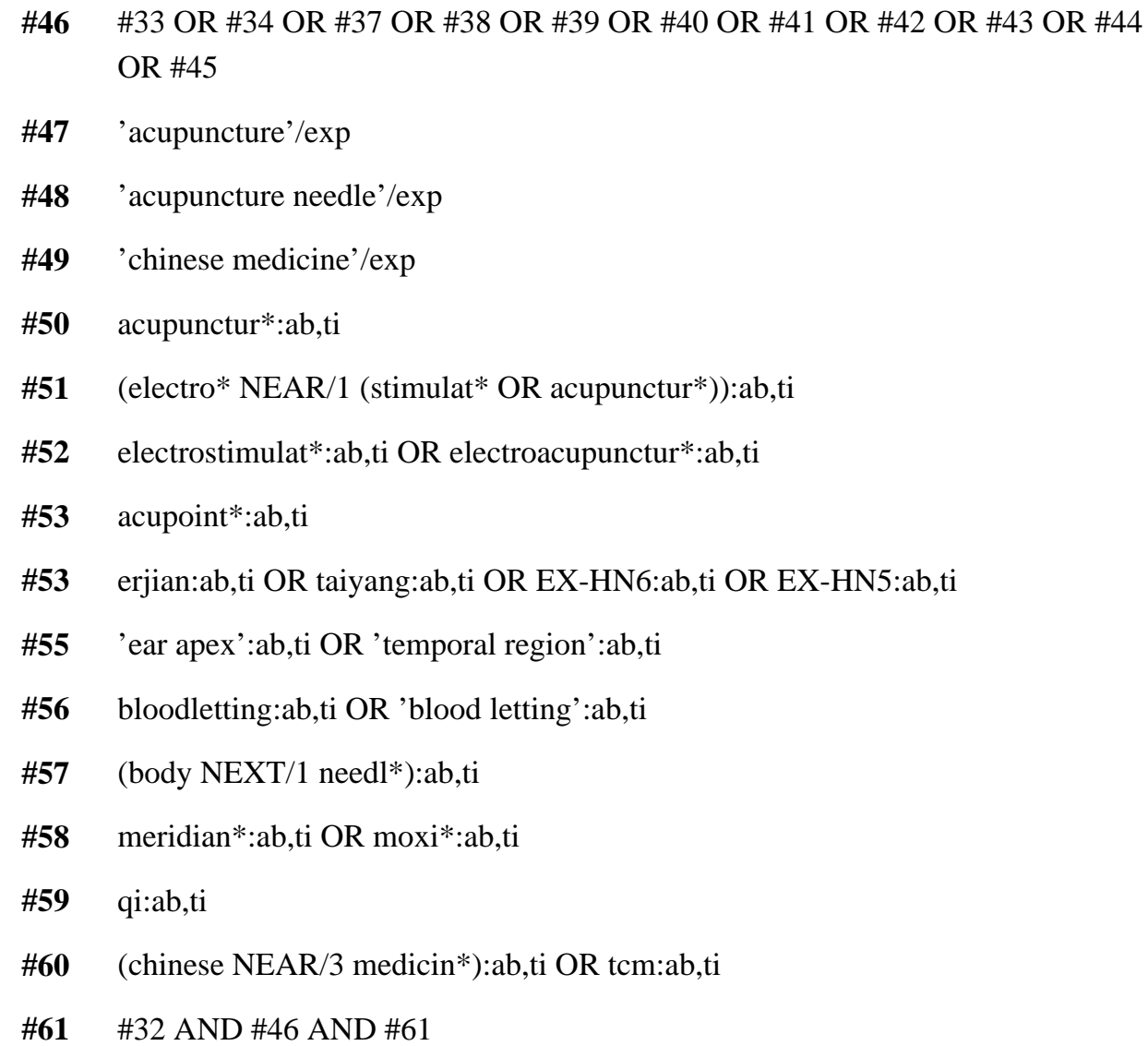

\section{Appendix 4. LILACS search strategy}

(Hordeol\$ OR Orzuelo OR Terçol OR Stye\$ OR MH:C01.252.354.400\$ OR MH:C01.539.375.354.400\$ OR MH: C11.294.354.400\$ OR MH:C11.338.648\$ OR Meibomian OR Tarsal OR Palpebral OR Conjunctiv\$ OR Zeis\$ OR Moll\$ OR “Glándulas Tarsales" OR "Glândulas Tarsais” OR MH:A09.371.337.614\$ OR MH:A10.336.827.600\$) AND (Acupunctur\$ OR MH:H02.004\$ OR MH:HP3.018.069\$ OR MH:E02.190.044\$ OR Chinese Traditional Medicine OR TCM OR "Medicina China Tradicional" OR "Medicina Tradicional Chinesa" OR “Zhong Yi Xue” OR "Chung I Hsueh” OR MH:E02.190.488.585.520\$ OR MH:I01.076.201.450.654.558.520\$ OR Bloodletting OR Venodisección OR Sangria OR MH:E02.800.558.500\$ OR Meridian\$ OR moxi\$ OR electrostimulat\$ OR electroacupunctur\$ OR electro-stimulat\$ OR electro-acupunctur\$ OR acupoint\$ OR erjian OR taiyang OR EX-HN6 OR EX-HN5 OR "body needling” OR bloodletting OR blood-letting OR Ear-apex OR “temporal region” OR qi)

\section{Appendix 5. PubMed search strategy}

\#1 ((randomized controlled trial[pt]) OR(controlled clinical trial[pt]) OR (randomised[tiab] OR randomized[tiab]) OR (placebo[tiab]) OR (drug therapy[sh]) OR (randomly[tiab]) OR (trial[tiab]) OR (groups[tiab])) NOT (animals[mh] NOT humans[mh]) 
\#2 (Meibomian*[tiab] AND (gland*[tiab] OR cyst*[tiab] OR inflammat*[tiab] OR infection*[tiab])) NOT Medlines[sb]

\#3 (tarsal[tiab] AND (gland*[tiab] OR cyst*[tiab] OR inflammat*[tiab] OR infection*[tiab])) NOT Medline[sb]

\#4 (palpebral[tiab] AND (gland*[tiab] OR cyst*[tiab] OR inflammat*[tiab] OR infection*[tiab])) NOT Medline[sb]

\#5 (conjunctiv*[tiab] AND (gland*[tiab] OR cyst*[tiab])) NOT Medline[sb]

\#6 (gland*[tiab] AND (zeis*[tiab] OR Moll*[tiab])) NOT Medline[sb]

\#7 ((lid*[tiab] OR eyelid*[tiab] OR eye margin[tiab]) AND inflammat*[tiab]) NOT Medline[sb]

\#8 ((lid*[tiab] OR eyelid*[tiab] OR eye margin[tiab]) AND infection*[tiab]) NOT Medline[sb]

\#9 \#2 OR \#3 OR \#4 OR \#5 OR \#6 OR \#7 OR \#8

\#10 acupunctur*[tiab] NOT Medline[sb]

\#11 (meridian*[tiab] OR moxi*[tiab]) NOT Medline[sb]

\#12 (electrostimulat*[tiab] OR electroacupunctur*[tiab] OR electro-stimulat*[tiab] OR electro-acupunctur*[tiab]) NOT Medline[sb]

\#13 acupoint*[tiab] NOT Medline[sb]

\#14 (erjian[tiab] OR taiyang[tiab] OR EX-HN6[tiab] OR EX-HN5[tiab]) NOT Medline[sb]

\#15 body needl*[tiab] NOT Medline[sb]

\#16 (bloodletting[tiab] OR blood-letting[tiab]) NOT Medline[sb]

\#17 (Ear-apex[tiab] OR temporal region[tiab]) NOT Medline[sb]

\#18 Qi[tiab] NOT Medline[sb]

\#19 ((Chinese[tiab] AND medicin*[tiab]) OR TCM[tiab]) NOT Medline[sb]

\#20 \#10 OR \#11 OR \#12 OR \#13 OR \#14 OR \#15 OR \#16 OR \#17 OR \#18 OR \#19

\#21 \#1 AND \#9 AND \#20

\section{Appendix 6. Chinese CNKI Database search strategy}

Strategy in Chinese phonetic alphabet (i.e., Pinyin):

(SU='Mai li zhong' or SU='Jian xian yan' or SU='Zhen yan') and (SU='Zhen ci' or $\mathrm{SU}=$ 'Zhen jiu' or SU='Fang xue' or SU='Ci luo' or SU='Er zhen' or $\mathrm{SU}=$ 'Ci xue' or $\mathrm{SU}=$ 'Tiao ci' or SU='Dian ci' or SU='Er ya' or SU='Er xue')

$\mathrm{SU}=$ subject, includes title, abstract and key words; FT=full text. 
(SU='麦粒肿' or SU='睑腺炎' or SU='针眼') and (SU='针刺' or SU='针炎' or SU='放血' or SU='刺络' or SU='耳针' or SU='刺血' or SU='挑刺' or SU='点刺' or SU='耳压' or SU='耳穴')

SU =subject 主题

\section{Appendix 7. SinoMed Database search strategy}

Strategy in Chinese phonetic alphabet (i.e., Pinyin):

(Mai li zhong OR Jian xian yan OR Zhen yan) AND (Zhen ci OR Zhen jiu OR Fang xue OR Ci luo OR Er zhen OR Ci xue OR Tiaoci OR Dian ci OR Er ya OR Er xue)

--- search in Que sheng (Que sheng includes title, MeSH, keywords, abstract, journal name) (麦粒肿 or 睑腺炎 or 针眼) and (针刺 or 针炎 or 放血 or 刺络 or 耳针 or 刺血 or 挑刺 or 点刺 or 耳压 or 耳穴) 在缺省里检索

\section{Appendix 8. Chinese VIP Database search strategy}

Strategy in Chinese phonetic alphabet (i.e., Pinyin):

$\mathrm{U}=($ Mai li zhong+Jian xian yan+Zhen yan)*(Zhen ci+Zhen jiu+Fang xue+Ci luo+Er zhen $+\mathrm{Ci}$ xue+Tiao ci+Dian ci+Er ya+Er xue)

$\mathrm{U}$ means all text.

$\mathrm{U}=($ 麦粒肿+睑腺炎 + 针眼 $) *($ 针刺 + 针炎 + 放血+刺络 + 耳针+刺血+挑刺 + 点刺 + 耳压 + 耳穴 $)$

\section{Appendix 9. metaRegister of Controlled Trials search strategy \\ Hordeolum OR stye}

\section{Appendix 10. ClinicalTrials.gov search strategy}

Hordeolum OR stye

\section{Appendix 11. ICTRP search strategy \\ Hordeolum OR stye}

\section{REFERENCES}

\section{Additional references}

* Indicates the major publication for the study

Adams D, Wu T, Yasui Y, Aung S, Vohra S. Systematic reviews of TCM trials: how does inclusion of Chinese trials affect outcome? Journal of Evidence-based Medicine. 2012; 5(2):89-97. [PubMed: 23557472]

Barza M, Baum J. Ocular infections. The Medical Clinics of North America. 1983; 67(1):131-152. [PubMed: 6338305] 
Bessette, M. [accessed 15 Sep 2013] Hordeolum and stye in emergency medicine. http:// emedicine.medscape.com/article/798940-overview

Bharathi MJ, Ramakrishnan R, Shivakumar C, Meenakshi R, Lionalraj D. Etiology and antibacterial susceptibility pattern of community-acquired bacterial ocular infections in a tertiary eye care hospital in south India. Indian Journal of Ophthalmology. 2010; 58(6):497-507. [PubMed: 20952834]

Cheng, XN. Chinese Acupuncture and Moxibustion. Beijing: Foreign Languages Press; 2010.

Cherkin DC, Sherman KJ, Deyo RA, Shekelle PG. A review of the evidence for the effectiveness, safety, and cost of acupuncture, massage therapy, and spinal manipulation for back pain. Annals of Internal Medicine. 2003; 138(11):898-906. [PubMed: 12779300]

Chern, KC.; Saidel, M. Ophthalmology Review Manual. 2nd Edition. Philadelphia: Lippincott Williams and Wilkins; 2011 Sep 20.

Deeks, JJ.; Higgins, JPT.; Altman, DG., editors; Higgins, JPT.; Green, S., editors. Chapter 9: Analysing data and undertaking meta-analyses. Cochrane Handbook for Systematic Reviews of Interventions Version 5.1.0. (updated March 2011). Available from www.cochrane-handbook.org

Deibel JP, Cowling K. Ocular inflammation and infection. Emergency Medicine Clinics of North America. 2013; 31(2):387-397. [PubMed: 23601478]

Ehrenhaus, M. [accessed 15 Sep 2013] Hordeolum. http://emedicine.medscape.com/article/1213080overview

Garrity, J. [accessed 15 Sep 2013] Chalazion and hordeolum. www.merckmanuals.com/professional/ eye_disorders/eyelid_and_lacrimal_disorders/chalazion_and_hordeolum.html

Gerstenblith, AT.; Rabinowitz, MP. The Wills Eye Manual: Office and Emergency Room Diagnosis and Treatment of Eye Disease. 6th Edition. Philadelphia: Lippincott Williams and Wilkins; 2012 Mar 19.

Grant, G. [accessed 15 Sep 2013] Sty. www.emedicinehealth.com/sty/article_em.htm

Han JS, Tang J, Ren MF, Zhou ZF, Fan SG, Qiu XC. Central neurotransmitters and acupuncture analgesia. The American Journal of Chinese Medicine. 1980; 8(4):331-348. [PubMed: 6113756]

Han JS. Acupuncture: neuropeptide release produced by electrical stimulation of different frequencies. Trends in Neurosciences. 2003; 26(1):17-22. [PubMed: 12495858]

Chapter 8: Assessing risk of bias in included studies. In: Higgins, JPT.; Altman, DG.; Sterne, JAC., editors; Higgins, JPT.; Green, S., editors. Cochrane Handbook for Systematic Reviews of Interventions Version 5.1.0. (updated March 2011). Available from www.cochrane-handbook.org

Higgins, JPT.; Deeks, JJ.; Altman, DG., editors; Higgins, JPT.; Green, S., editors. Chapter 16: Special topics in statistics. Cochrane Handbook for Systematic Reviews of Interventions Version 5.1.0. (updated March 2011). Available from www.cochrane-handbook.org

Hsiao CH, Chuang CC, Tan HY, Ma DH, Lin KK, Chang CJ, et al. Methicillin-resistant Staphylococcus aureus ocular infection: a 10-year hospital-based study. Ophthalmology. 2012; 119(3):522-527. [PubMed: 22176801]

Law SK, Li T. Acupuncture for glaucoma. Cochrane Database of Systematic Reviews. 2013; (Issue 5)

Lederman C, Miller M. Hordeola and chalazia. Pediatrics in Review. 1999; 20(8):283-284. [PubMed: 10429150]

Lee MS, Shin BC, Choi TY, Ernst E. Acupuncture for treating dry eye: a systematic review. Acta Ophthalmologica. 2011; 89(2):101-106. [PubMed: 20337604]

Li A, Lao L, Wang Y, Xin J, Ren K, Berman BM, et al. Electroacupuncture activates corticotrophinreleasing hormone-containing neurons in the paraventricular nucleus of the hypothalammus to alleviate edema in a rat model of inflammation. BMC Complementary and Alternative Medicine. 2008; 8:20. [PubMed: 18474100]

Lindsley K, Nichols JJ, Dickersin K. Interventions for acute internal hordeolum. Cochrane Database of Systematic Reviews. 2013; (Issue 4)

MacPherson H, White A, Cummings M, Jobst K, Rose K, Niemtzow R, et al. Standards for reporting interventions in controlled trials of acupuncture: the STRICTA recommendations. Complementary Therapies in Medicine. 2001; 9(4):246-249. [PubMed: 12184354] 
Man PL, Chen CH. Mechanism of acupunctural anesthesia. The two-gate control theory. Diseases of the Nervous System. 1972; 33(11):730-735. [PubMed: 4347637]

Manheimer E, Cheng K, Linde K, Lao L, Yoo J, Wieland S, et al. Acupuncture for peripheral joint osteoarthritis. Cochrane Database of Systematic Reviews. 2010; (Issue 1)

Melchart D, Weidenhammer W, Streng A, Reitmayr S, Hoppe A, Ernst E, et al. Prospective investigation of adverse effects of acupuncture in 97733 patients. Archives of Internal Medicine. 2004; 164(1):104-105. [PubMed: 14718331]

Moffet HH. How might acupuncture work? A systematic review of physiologic rationales from clinical trials. BMC Complementary and Alternative Medicine. 2006; 6:25. [PubMed: 16824230]

Mueller JB, McStay CM. Ocular infection and inflammation. Emergency Medicine Clinics of North America. 2008; 26(1):57-72. [PubMed: 18249257]

Peralejo B, Beltrani V, Bielory L. Dermatologic and allergic conditions of the eyelid. Immunology and Allergy Clinics of North America. 2008; 28(1):137-168. [PubMed: 18282550]

The Nordic Cochrane Centre, The Cochrane Collaboration. Review Manager. 5.2. Copenhagen: The Nordic Cochrane Centre, The Cochrane Collaboration; 2013.

Schünemann, HJ.; Oxman, AD.; Vist, GE.; Higgins, JPT.; Deeks, JJ.; Glasziou, P., et al. Higgins, JPT.; Green, S., editors. Chapter 12: Interpreting results and drawing conclusions. Cochrane Handbook for Systematic Reviews of Interventions Version 5.1.0. (updated March 2011). Available from www.cochrane-handbook.org

Shen, XY.; Wang, H. Acupuncture and Moxibustion. 2nd Edition. Beijing: People's Medical Publishing House; 2007 Sep.

Skorin, L. [accessed 4 June 2008] Hordeolum and chalazion treatment: the full gamut. www.optometry.co.uk/clinical/details?aid=247

Tasman, W.; Jaeger, EA. Duane's Ophthalmology on DVD-ROM. 2013 Edition. Philadelphia: Lippincott Williams and Wilkins; 2012 Dec 27.

Wald ER. Periorbital and orbital infections. Infectious Disease Clinics of North America. 2007; 21(2): 393-408. [PubMed: 17561075]

Wei ML, Liu JP, Li N, Liu M. Acupuncture for slowing the progression of myopia in children and adolescents. Cochrane Database of Systematic Reviews. 2011; (Issue 9)

White A, Hayhoe S, Hart A, Ernst E. Adverse events following acupuncture: prospective survey of 32 000 consultations with doctors and physiotherapists. British Medical Journal. 2001; 323(7311): 485-486. [PubMed: 11532840]

World Health Organization. A proposed standard international acupuncture nomenclature: report of a WHO scientific group. Geneva: World Health Organization; 1991. A proposed standard international acupuncture nomenclature: report of a WHO scientific group.

World Health Organization. Acupuncture: review and analysis of reports on controlled clinical trials. Geneva: World Health Organization; 2003.

Witt CM, Pach D, Brinkhaus B, Wruck K, Tag B, Mank S, et al. Safety of acupuncture: results of a prospective observational study with 229,230 patients and introduction of a medical information and consent form. Forschende Komplementarmedizin. 2009; 16(2):91-97. [PubMed: 19420954]

Wu T, Li Y, Bian Z, Liu G, Moher D. Randomized trials published in some Chinese journals: how many are randomized? Trials. 2009; 10:46. [PubMed: 19573242]

Xu S, Wang L, Cooper E, Zhang M, Manheimer E, Berman B, et al. Adverse events of acupuncture: a systematic review of case reports. Evidence-based Complementary and Alternative Medicine : ECAM. 2013; 2013:581203. [PubMed: 23573135] 


\section{SOURCES OF SUPPORT}

\section{Internal sources}

- No sources of support supplied

\section{External sources}

- National Basic Research Program of China, China.

Ke Cheng and Xueyong Shen were funded by the National Basic Research Program of China (2009CB522901, 2012CB518502), the Key Program of State Administration of Traditional Chinese Medicine of China and the Shanghai Municipal Science Foundation (11DZ1973300, ZYSNXD-CC-ZDXK-07), and National Natural Science Foundation of China (81320108028).

- Key Program of State Administration of Traditional Chinese Medicine of China, the Shanghai Municipal Science Foundation, and 2014 Innovation Program of the Shanghai Municipal First-Class Field of Traditional Chinese Medicine, China.

Ke Cheng and Xueyong Shen were funded by the National Basic Research Program of China (2009CB522901, 2012CB518502), the Key Program of State Administration of Traditional Chinese Medicine of China and the Shanghai Municipal Science Foundation (11DZ1973300, ZYSNXD-CC-ZDXK-07), and National Natural Science Foundation of China (81320108028).

- National Natural Science Foundation of China, China.

Ke Cheng and Xueyong Shen were funded by the National Basic Research Program of China (2009CB522901, 2012CB518502), the Key Program of State Administration of Traditional Chinese Medicine of China and the Shanghai Municipal Science Foundation (11DZ1973300, ZYSNXD-CC-ZDXK-07), and National Natural Science Foundation of China (81320108028).

- National Eye Institute, National Institutes of Health, USA.

Xue Wang is supported by the Cochrane Eyes and Vision Group US Project through the National Eye Institute, Grant 1 U01 EY020522-01

- National Center for Complementary and Alternative Medicine (NCCAM), National Institutes of Health, USA. Lixing Lao was partially funded by Grant Number R24 AT001293

- National Center for Complementary and Alternative Medicine (NCCAM) of the US National Institutes of Health, USA. L. Susan Wieland was partially funded by Grant Number R24 AT001293 